\section{Gospelisation: a condition affecting management protocols}

Sir,

Increasing complexity of medical technology leads to more reliance on management protocols, with reduced opportunities for the users to check their scientific foundations. The term 'gospelisation' is proposed to describe a process illustrated by the following example:

A clinical neonatal computer database (to be described elsewhere) included a daily record of babies' actual fluid intake, in $\mathrm{ml} / \mathrm{kg} /$ day. Several very low birthweight infants were shown to have been receiving regularly between 200 and $250 \mathrm{ml} / \mathrm{kg} /$ day, all or mostly intravenously, although it had been thought that $200 \mathrm{ml} / \mathrm{kg} /$ day was the upper limit of intravenous fluid administered.

The explanation was that the intravenous feeding protocol contained an instruction to feed to an 'expected' weight, which often exceeded the actual weight of the baby. This instruction was apparently inherited from an earlier set of guidelines for milk fed babies which had been produced for a previous intravenous feeding regimen. The protocol had assumed the status of invariable unit policyhad become 'gospelised'-over the years.

It is suggested that management protocols are vulnerable to gospelisation, especially if they are technically complex, are based on an eclectic selection of evidence, or their elements are interdependent so that one element cannot be changed without reviewing the whole protocol. Their practical application to patients should be monitored, and clinical computer databases may make this easier.

\section{Fathers are easily worried too}

Sir,

With reference to the otherwise excellent article by the Illingworths entitled 'Mothers are easily worried', 'may I point out that so are fathers! May I put in a plea for paediatricians to continue to recognise the important part that fathers play in families, even though they do not necessarily always attend the outpatients clinic.

F Sheehy Skeffington Barnsley District General Hospital, Barnsley S75 2EP

\section{Reference}

1 Illingworth CA, Illingworth RS. Mothers are casily worried. Arch Dis Child 1984;59:380)-4.

B LASK (psychiatrist and father) The Hospitals for Sick Children, Great Ormond Street, London WCIN $3 \mathrm{JH}$

\title{
Correction
}

There is an error in the Correspondence section of the May issue of the Archives. 'Haemophilia and T lymphocyte subsets' p 491, para 5. line 2, for 'antibody' please read 'antigen'. The authors would like to point out that hepatitis B surface antibody was in fact positive in four of their patients. 